15 years, were reviewed. 11 patients were neuroblastoma, 3 were nonhodgkin lymphoma and the last were rhabdomyosarcoma. Male/female ratio was 1.2, the median age was 5.2 years, 9 patients went to surgery for tumour resection and laminectomy, others were treated with chemotherapy after biopsy. After treatment, 3 of severely affected 6 patients, who presented with paralysis, completely recovered neurologic function. 2 of 4 patients with moderate deficits, consisting of paresis and bowel/bladder dysfunction, completely recovered neurologic function. 7 children, who had mild symptoms comprised of paresis alone, fully recovered.

The frequency of complete neurologic recovery in children with intraspinal mass inversely correlated with the severity of the presenting neurologic deficits. The time passed between the first symptom and treatment was also important for neurologic recovery.

\section{ANALYSIS OF SURVIVIN GENE POLYMORPHISM AND SURVIVIN EXPRESSION IN WILMS TUMOURS IN SERBIAN CHILDREN}

doi:10.1136/archdischild-2012-302724.1468

'S Radojevic-Skodric, 'G Basta-Jovanovic, 'D Brasanac, ${ }^{2} \mathrm{~N}$ Nikolic, 'L Bogdanovic, ${ }^{2} \mathrm{~J}$ Milasin. 'Institute of Pathology, School of Medicine, University of Belgrade; 'Institute of Human Genetics, School of Dentistry, University of Belgrade, Belgrade, Serbia

Survivin, an apoptotic inhibitor, is overexpressed in various types of cancer. It has been shown that single nucleotide polymorphisms in the survivin gene promoter can modulate survivin expression and consequently influence the risk for some cancers. The aims of the present study were to:

a. analyze by means of PCR/RFLP, genotype and allele frequencies for the promoter $-31 \mathrm{G} / \mathrm{C}$ polymorphism in the survivin gene of 59 Wilms tumour (WT) patients and 82 controls,

b. determine cytoplasmic and nuclear survivin expression in WTs using immunohistochemical methods.

The frequencies of alleles and genotypes were significantly different between patients and controls for the $-31 \mathrm{G} / \mathrm{C}$ polymorphism. Individuals with CC and CG genotypes had significantly decreased risk of WT compared to GG individuals (OR 0.26, 95\% CI 0.07-0.96; OR $0.30,95 \%$ CI $0.15-0.60)$. A statistically significant difference in cytoplasmic survivin expression between lower and higher grades tumours has been detected as well $(p=0.000)$, but without correlation with the genotypes. Our findings suggest that both survivin genotypes and survivin expression, though not showing direct relationship, represent relevant risk/prognostic markers for WT in Serbian population.

\section{YEARS CASE REVIEW ON HEPATOBLASTOMA IN NORTHERN IRELAND}

doi:10.1136/archdischild-2012-302724.1469

'VK Sundarajan, ${ }^{2} \mathrm{~A}$ McCarthy. ' Specialty Registrar in Paediatrics; ${ }^{2}$ Paediatric Oncologist, Childrens Haematology/Oncology Unit, Royal Belfast Hospital for Sick Children, Belfast, UK

Aims To look at the incidence, management and outcome of Hepatoblastoma over 20 years period in Northern Ireland.

Methods A retrospective audit conducted in December 2011. Data collected from medical notes of all children diagnosed with Hepatoblastoma from Jan 1991 to Nov 2011 (14 patients), using an Audit proforma sheet.

Results Age at presentation: < 2 years-11 patients (79\%), 2 to 15 years-3 patients (21\%).

AFP level: High in all patients ranging from 12,589 to $3,140,000$ $\mathrm{KU} / \mathrm{L}$

Platelet count: Abnormally high-8 patients (57\%), Normal-6 patients (43\%). LFT: Deranged-11 patients (79\%), Normal-3 patients(21\%).
Clotting screen: Normal-11 patients (79\%), Deranged-2 patients (15\%), Not performed-1 patient $(7 \%)$.

Staging: Pretext II-7 patients (51\%), Pretext III-3 patients $(21 \%)$, Pretext IV-3 patients (21\%), Awaited-1 patient (7\%).

Diagnosis: Non Metastatic Hepatoblastoma-Standard risk 8 patients (58\%) and High risk 3 patients (21\%),Metastatic Hepatoblastoma 3 patients (21\%).

Pre-op chemotherapy: SIOPEL one-1 patient (7\%),SIOPEL three-8 patients $(57 \%)$, SIOPEL four-5 patients $(36 \%)$.

Chemotherapy treatment: On time-11 patients (79\%), Delayed-3 patients (21\%).

Timing of surgery from initial diagnosis: 10 patients (70\%) 2-4 months, 2 patients (15\%) 4-5 months, 2 patients (15\%) awaiting.

Type of Surgery: Orthotopic liver transplant-3 patients (21\%), Partial hepatectomy-9 patients (64\%), Awaiting-2 patients (15\%).

Associated syndrome: Present (Beckwith-Wiedemann syndrome, Fanconi syndrome and 2 parents had familial polyposis coli)-4 patients (30\%), Absent-10 patients (70\%).

Post-op chemotherapy: SIOPEL one-1 patient (7\%), SIOPEL three-7 patients $(50 \%)$, SIOPEL four-3 patients (21\%), Not applicable-1 patient $(7 \%)$, Awaited-2 patients (15\%).

Complications of chemotherapy: Yes (Hearing loss)-3 patients (21\%), Nil-11 patients (79\%).

Outcome: Remission-10 patients (70\%), Died-2 patients $(15 \%)$, Undergoing treatment-2 patients (15\%).

\section{Conclusions}

Incidence Overall 0.02 per 100,000 versus 2 per 100,000 worldwide.

Platelet count: Abnormally high in $57 \%$ patients.

Survival rate: Overall 83.5\% (10/12) versus 70\% worldwide.

\section{ESTIMATION OF CIRCULATING SVCAM-1, SESELECTIN AND SIL2R IN CHILDREN WITH ACUTE LYMPHOBLASTIC LEUKEMIA}

doi:10.1136/archdischild-2012-302724.1470

'S Kholoussi, ${ }^{2} \mathrm{~A}$ Khorshed. 'Immunogenetics, Human Genetics, National Research Center; ${ }^{2}$ Hematology, National Cancer Institute, Cairo, Egypt

Background Measurement of some circulating endothelial adhesion molecules and soluble receptors levels have been suggested as additional tools for assessment of patients with acute lymphoblastic leukemia (ALL). The aim of this study is to estimate sVCAM-1, sEselectin and sIL2R levels in the serum of cases with childhood acute lymphoblastic leukemia, comparing their levels before and after chemotherapy and comparing them to the control group. Moreover patients were categorised into responder and non-responder.

Patients and Method This study included ninety subjects attending National Cancer Institute; Twenty newly diagnosed patients with acute lymphoblastic leukemia (ALL) before starting chemotherapy, 40 patients with ALL after chemotherapy. 10 patients in partial remission and 20 apparently normal subjects within the same age and sex range, as a control group. Acute lymphoblastic leukemia diagnosis was made by bone marrow aspiration, cytochemistry and microscopic examination. Flowcytomer was used for immunophenotyping to confirm the diagnosis using monoclonal antibodies. SVCAM-1, sEselectin and sIL2R were estimated using enzyme-linked immunosorbent assay.

Results There were significant increases in the three estimated parameters in patients before chemotherapy as compared to after treatment as well as to the control group.

Conclusion The levels of some soluble circulating adhesion molecule and soluble receptors levels can be utilized for monitoring the disease activity of ALL and its response to treatment. 